\title{
Cushing's syndrome: a rare cause of multiple vertebral fractures shortly after a complicated pregnancy
}

Yu Ji Kim and Kyung Ae Lee

Division of Endocrinology and Metabolism, Department of Internal Medicine, Research Institute of Clinical Medicine of Jeonbuk National University-Biomedical Research Institute of Jeonbuk National University Hospital, Jeonju, Korea

Received : May 19, 2021

Revised : June 13, 2021

Accepted: June 24, 2021

\section{Correspondence to} Kyung Ae Lee, M.D. Tel: $+82-63-250-2749$ Fax: +82-63-250-2747 E-mail: kaleey@jbnu.ac.kr https://orcid.org/0000-00033700-8279
A 28-year-old woman visited the endocrinology department with a chief complaint of back pain after falling. Four months ago, she had preeclampsia and gestational diabetes mellitus, which resulted in the premature delivery of her second baby at 29 weeks of gestation. Her first baby was also delivered prematurely at 35 weeks of gestation 1 year ago. The patient's height, weight, and blood pressure were $155 \mathrm{~cm}, 63 \mathrm{~kg}$, and 148/100 $\mathrm{mmHg}$, respectively. Additionally, truncal obesity and purple abdominal striae were observed. On radiography, multiple compression fractures were observed in the thoracic and lum- bar vertebrae. In particular, T9, T10, T12, and L1 to L4 were fractured (Fig. 1A). The bone mineral density of the lumbar spine could not be evaluated owing to a fracture. Her hip Z-score was -1.9 (Fig. 1B). Contrast enhanced computed tomography scan of the abdomen was conducted to reveal a mass measuring $2.8 \times 2.0 \mathrm{~mm}$ in size on the right adrenal gland (Fig. 2). Hormonal tests of the adrenal gland were performed, and the results were as follows: $0.9 \mathrm{ng} / \mathrm{mL} / \mathrm{hr}$ plasma renin activity, $5.7 \mathrm{ng} / \mathrm{dL}$ aldosterone, 6.3 ratio of aldosterone to renin, 0.09 $\mathrm{nmol} / \mathrm{L}(<0.50)$ plasma metanephrine, $28.4 \mu \mathrm{g} / \mathrm{dL}$ serum cortisol, $1.37 \mathrm{pg} / \mathrm{mL}$
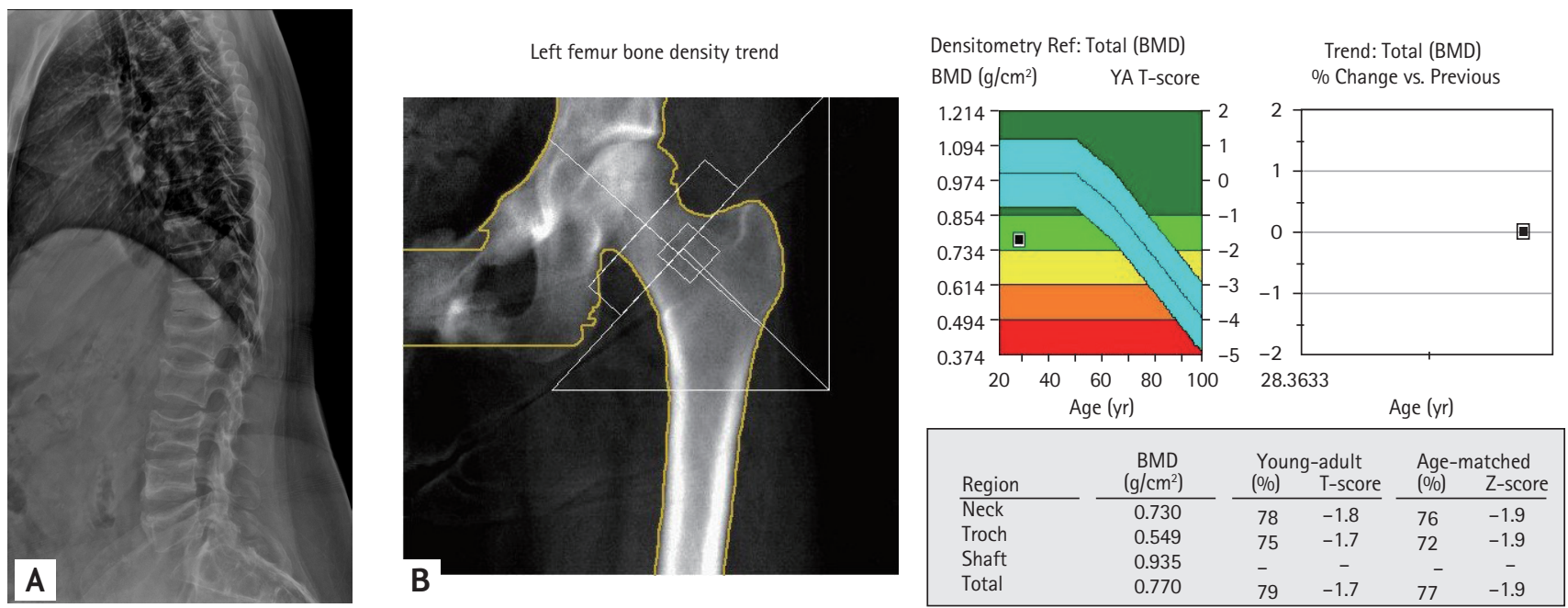

Figure 1. Spine radiography and bone mineral density of the patient. (A) On thoracolumbar spine radiography, multiple compression fractures were observed in the thoracic and lumbar vertebrae. In particular, T9, T10, T12, and L1 to L4 were involved. (B) Bone mineral density (BMD) of the lumbar spine could not be evaluated owing to the presence of a fracture. The hip Z-score was -1.9. 


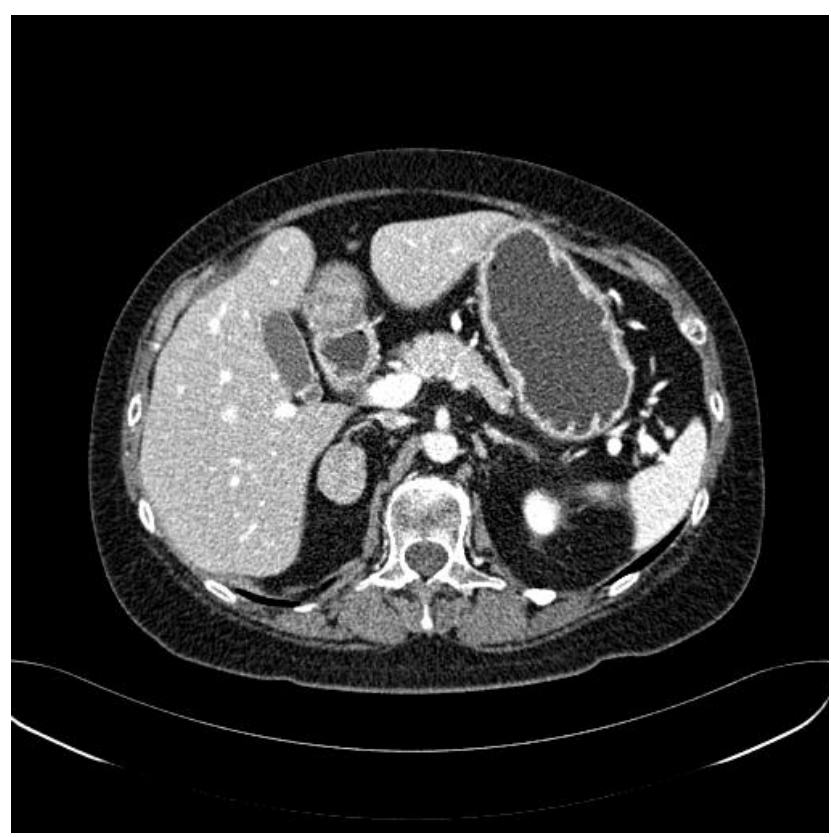

Figure 2. Spiral computed tomography (CT) scan of abdomen. The contrast-enhanced CT scan shows a mass that is approximately $2.8 \times 2.0 \mathrm{~cm}$ in size in the right adrenal gland.

adrenocorticotropic hormone (ACTH), and overnight 1-mg dexamethasone suppressed cortisol of $28.6 \mu \mathrm{g} / \mathrm{dL}$
(<1.8). The results were compatible with ACTH-independent Cushing's syndrome (CS). Weekly alendronate with daily calcium and cholecalciferol replacement therapy was initiated for osteoporosis. After a laparoscopic removal of the adrenal mass, her morning serum cortisol level decreased to $1.1 \mu \mathrm{g} / \mathrm{dL}$. Active CS during pregnancy is associated with a high rate of maternal and fetal complications. Pregnancy-associated CS is easy to overlook and remains a diagnostic challenge. This is because pregnancy itself is rare in CS patients. Various symptoms and signs of CS can also occur in relation to normal pregnancy. Furthermore, interpretation of diagnostic tests is also difficult. The predominant etiology of CS is adrenal adenoma, which is reported in $40 \%$ to $60 \%$ of cases. The present case demonstrates the importance of considering CS as a differential diagnosis in a patient who presents with multiple vertebral fractures, preeclampsia, or gestational diabetes mellitus, regardless of whether the patient is pregnant or postpartum.

\section{Conflict of interest}

No potential conflict of interest relevant to this article was reported. 